\title{
Increased activation of lymphocytes infiltrating primary colorectal cancers following immunisation with the anti-idiotypic monoclonal antibody 105AD7
} C A Maxwell-Armstrong, L G Durrant, R A Robins, A M Galvin, J H Scholefield,
J D Hardcastle
University

Department of

Surgery, Queen's

Medical Centre,

Nottingham NG7 2UH,

UK

C A Maxwell-Armstrong

L G Durrant

J H Scholefield

J D Hardcastle

University

Department of Clinical

Oncology, City

Hospital, Nottingham

NG5 1PB, UK

L G Durrant

Department of

Immunology,

University of

Nottingham,

Nottingham, UK

R A Robins

A M Galvin

Correspondence to:

Mr Maxwell-Armstrong.

Accepted for publication 18 March 1999

\begin{abstract}
Background-The anti-idiotypic monoclonal antibody 105AD7 mimics the tumour associated antigen 791Tgp72, expressed on $70-80 \%$ of colorectal cancers. Phase I studies have shown that the vaccine is non-toxic, and a number of patients have been immunised prior to resection of their primary tumours.

Aims-To assess lymphocyte activation at the tumour site by measuring expression of the $\alpha$ subunit of the interleukin 2 receptor (CD25).

Methods-Nineteen patients with primary colorectal cancer were immunised with varying doses of $105 \mathrm{AD} 7$ prior to resection of their primary tumours. Samples of normal bowel and tumour edge/centre from 16 patients were available for immunohistochemical staining with a monoclonal antibody against CD25. Samples from a matched control group were also stained. Fresh tumours from 14 immunised patients and 31 unimmunised control patients were disaggregated, and the lymphocytes obtained labelled for CD25. Samples were analysed blindly by flow cytometry.

Results-Median infiltration of lymphocytes expressing CD25, measured immunohistochemically, was higher in trial patients, as was the ratio of tumour to normal bowel infiltration. Flow cytometric analysis of fresh tumour from immunised patients showed a significantly higher percentage of lymphocytes expressing CD25 tumour infiltrating lymphocytes than their matched and unmatched controls.

Discussion-The $\alpha$ subunit of the interleukin 2 receptor is increased on tumour infiltrating lymphocytes, in patients immunised with the colorectal cancer vaccine 105AD7. This suggests a population of activated lymphocytes capable of targeting 791Tgp72 expressing tumour cells, such as circulating micrometastases. 105AD7 may have a role as adjuvant therapy in early stage disease. (Gut 1999;45:593-598)
\end{abstract}

Keywords: anti-idiotypic antibody; colorectal cancer; immunotherapy; 105AD7
Colorectal cancer accounts for approximately 16000 deaths per year in the UK. There has been little impact in overall survival in the last 30 years, ${ }^{12}$ renewing interest in immunotherapy as a potential adjuvant treatment. Advances in molecular pathology have meant that it is now possible to identify tumour associated antigens (TAA), such as 17-1A, carcinoembryonic antigen (CEA), and 791Tgp72, against which specific immune responses can be directed by vaccines, such as anti-idiotypic antibodies. $^{34}$

Patients with colorectal cancer injected with 791T/36 monoclonal antibody, directed against the TAA 791Tgp72, have been shown to generate an anti-idiotypic antibody, subsequently termed $105 \mathrm{AD} 7 .^{5}$ Complete blocking of 791T/36-fluorescein isothiocyanate (FITC) binding was seen in a quantitative flow cytometric assay, indicating that the antiidiotypic monoclonal antibody was mimicking the TAA. Cellular immune responses were seen in animal studies, ${ }^{6}$ and a phase I study in patients with advanced colorectal cancer has established that 105AD7 is non-toxic. ${ }^{7}$ In addition, patients receiving the vaccine lived for a median of 12 months - three times as long as a contemporary group. Nine of the 13 patients developed either an antigen specific $\mathrm{T}$ cell blastogenesis, or showed an increase in peripheral blood interleukin 2 (IL-2) concentrations, further suggesting that $\mathrm{T}$ cell responses could be generated. ${ }^{78}$

In view of the lack of toxicity, and accumulating evidence suggesting that advanced disease may not be the optimal setting in which to assess the efficacy of immunotherapeutic agents, ${ }^{9}$ further work concentrated on vaccinating patients with $105 \mathrm{AD} 7$ prior to resection of their primary tumours. CD8+ lymphocytes, CD4+ helper lymphocytes, and natural killer (NK) cells have been observed at the tumour site of those receiving 105AD7, and enhanced cytotoxicity has been shown against autologous tumour. ${ }^{10-12}$

Proliferation and differentiation of tumour infiltrating lymphocytes (TIL) is mediated by

Abbreviations used in this paper: FCS, fetal calf serum; FITC, fluorescein isothiocyanate; IL-2, interleukin 2; IQR, interquartile range; $\mathrm{MHC}$, major histocompatibility complex; NK, natural killer; PE, phycoerythrin; TAA, tumour associated antigen; TBS, Tris buffered saline; TIL, tumour infiltrating lymphocytes. 
Table 1 Demographics of patients receiving 105 AD7 prior to surgery

\begin{tabular}{|c|c|c|c|c|c|c|c|c|}
\hline $\begin{array}{l}\text { Patient } \\
\text { no }\end{array}$ & Sex & $\begin{array}{l}\text { Age at time of removal } \\
\text { of primary tumour }(y)\end{array}$ & $\begin{array}{l}\text { Site of } \\
\text { tumour }\end{array}$ & $\begin{array}{l}\text { Differentiation of } \\
\text { primary tumour }\end{array}$ & Dukes'stage & $\begin{array}{l}\text { Dose of } 105 A D 7 \\
(\mu g)\end{array}$ & $\begin{array}{l}\text { Immunohistochemical } \\
\text { analysis of tumour sections }\end{array}$ & $T I L$ \\
\hline 1 & Female & 75 & Rectum & Moderate & C & $10+50$ & Yes & Yes \\
\hline 2 & Female & 79 & Sigmoid & Moderate & B & $10+50$ & Yes & Yes \\
\hline 3 & Male & 82 & Caecum & Moderate & C & $10+50$ & Yes & Yes \\
\hline 4 & Male & 87 & Caecum & Moderate & B & $10+50$ & Yes & No \\
\hline 5 & Male & 75 & Rectum & Moderate & A & $10+50$ & Yes & Yes \\
\hline 6 & Male & 73 & Rectum & Moderate & C & $50+50$ & Yes & Yes \\
\hline 7 & Male & 70 & Rectum & Moderate & B & $50+50$ & Yes & No \\
\hline 8 & Male & 56 & Rectum & Moderate & B & $50+50$ & Yes & Yes \\
\hline 9 & Female & 67 & Rectum & Moderate & A & $50+50$ & Yes & Yes \\
\hline 10 & Female & 62 & Rectum & Poor & C & $50+50$ & Yes & Yes \\
\hline 11 & Male & 58 & Rectum & Moderate & A & $50+50$ & Yes & Yes \\
\hline 12 & Male & 76 & Sigmoid & Moderate & A & $50+50$ & Yes & Yes \\
\hline 13 & Female & 67 & Ascending & Poor & B & $10+100$ & Yes & No \\
\hline 14 & Male & 67 & Sigmoid & Moderate & A & $10+100$ & Yes & No \\
\hline 15 & Female & 82 & Rectum & Moderate & C & $10+100$ & Yes & No \\
\hline 16 & Male & 75 & Rectum & Moderate & D & $10+50^{\star}$ & Yes & Yes \\
\hline 17 & Male & 63 & Rectum & Moderate & C & $50+50$ & No & Yes \\
\hline 18 & Male & 75 & Rectum & Moderate & B & $10+20$ & No & Yes \\
\hline 19 & Female & 63 & Rectum & Moderate & B & $10+50$ & No & Yes \\
\hline
\end{tabular}

$\star$ Patient received two courses of this regimen prior to surgery.

TIL, tumour infiltrating lymphocytes.

IL-2 through a specific receptor. The cytokine is capable of upregulation of the receptor in a paracrine fashion, and its presence is consistent with $\mathrm{T}$ cell activation. ${ }^{13}$ The receptor consists of three subunits: $\alpha$ (CD25), $\beta$ (p70-75), and $\gamma$ (p64), the former being present in both high and low affinity receptors on CD4 and CD8 cells. ${ }^{14}$ This work shows by both flow cytometry and immunohistochemistry, that expression of the $\alpha$ subunit on TIL is increased in patients who have received 105AD7 preoperatively, and suggests a population of activated $T$ cells at the tumour site in these patients. This adds further strength to the hypothesis that $\mathrm{T}$ cells primed by $105 \mathrm{AD} 7$ in the periphery are capable of targeting 791Tgp72 antigen on colorectal cancer cells, whether they be at the site of the primary tumour as with this series of experiments, or more importantly on circulating micrometastases. The non-toxic vaccine 105AD7 may have a role in early stage colorectal cancer.

\section{Materials and methods}

PATIENTS

Nineteen patients with sporadic primary colorectal cancer, diagnosed either on biopsy or double contrast barium enema, were recruited preoperatively. The group consisted of 12 men and seven women, with a mean age of 71.2 years (range 56-87 years). Thirteen primary tumours were rectal, three sigmoid, two caecal, and one in the ascending colon (table 1).

CLINICAL PROTOCOL

This study was performed under the auspices of the Cancer Research Campaign UK Phase I/II clinical trials committee, and local ethical committee approval was obtained. Clinical grade monoclonal antibody was produced as previously described, ${ }^{16}$ and all patients gave informed consent to treatment with 105AD7.

Nineteen patients with primary colorectal cancer were immunised with $105 \mathrm{AD} 7$ prior to surgery. Varying dosing schedules and routes of administration were used as part of ongoing work seeking to optimise vaccination schedules. The mean time between first injection and resection of primary tumour was 16.8 days (range 9-26).
Specimens were retrieved and samples taken from the tumour centre, and one or two tumour edges, depending on sample size. A specimen of normal bowel, at least $20 \mathrm{~cm}$ away from the lesion, was also taken. All tumour tissue was confirmed as adenocarcinoma by routine histopathological examination.

\section{CONTROL GROUP}

Samples of tumour from all colorectal cancers removed at the Queen's Medical Centre over a three year period ending October 1996, were taken and cryopreserved in liquid nitrogen. These patients had not received 105AD7 prior to surgery. From this large bank of tumours it was possible to match each tumour specimen from patients who had received 105AD7 preoperatively to a control tumour according to site, stage, and differentiation of tumour, as well as sex and approximate age of patient. Both trial and control tumours were identically immunohistochemically labelled.

Fresh tumour was obtained from 31 patients with colorectal cancer, who had not received 105AD7 preoperatively. These acted as controls for the flow cytometric analysis. This group included 16 men and 15 women, with a mean age of 68.1 years. The majority of tumours were either caecal (11) or rectal (10), with the remainder being sigmoid colon (four), ascending colon (three), rectosigmoid (one), splenic flexure (one), and colon (1).

\section{IMMUNOHISTOCHEMISTRY}

Cryopreserved samples from 16 patients who had received $105 \mathrm{AD} 7$ were available for immunohistochemical analysis, and matched to unimmunised cryopreserved tumour specimens from unimmunised control tumours, as described. Tissue was cut into $3 \times 3 \mathrm{~mm}, 5 \mu \mathrm{m}$ thick sections, mounted on Vectabond (Vector Laboratories) coated slides, and acetone fixed. Sections were rehydrated and blocked using $20 \%$ rabbit serum, prior to staining with murine CD25 monoclonal antibody (Dako A/S, Produktionsvej 42 DK-2600, Glostrup, Denmark; $1 / 10$ dilution). Murine $\operatorname{IgG}_{1}$, similarly diluted, was used as a negative control. A standard avidin-biotin complex method was 

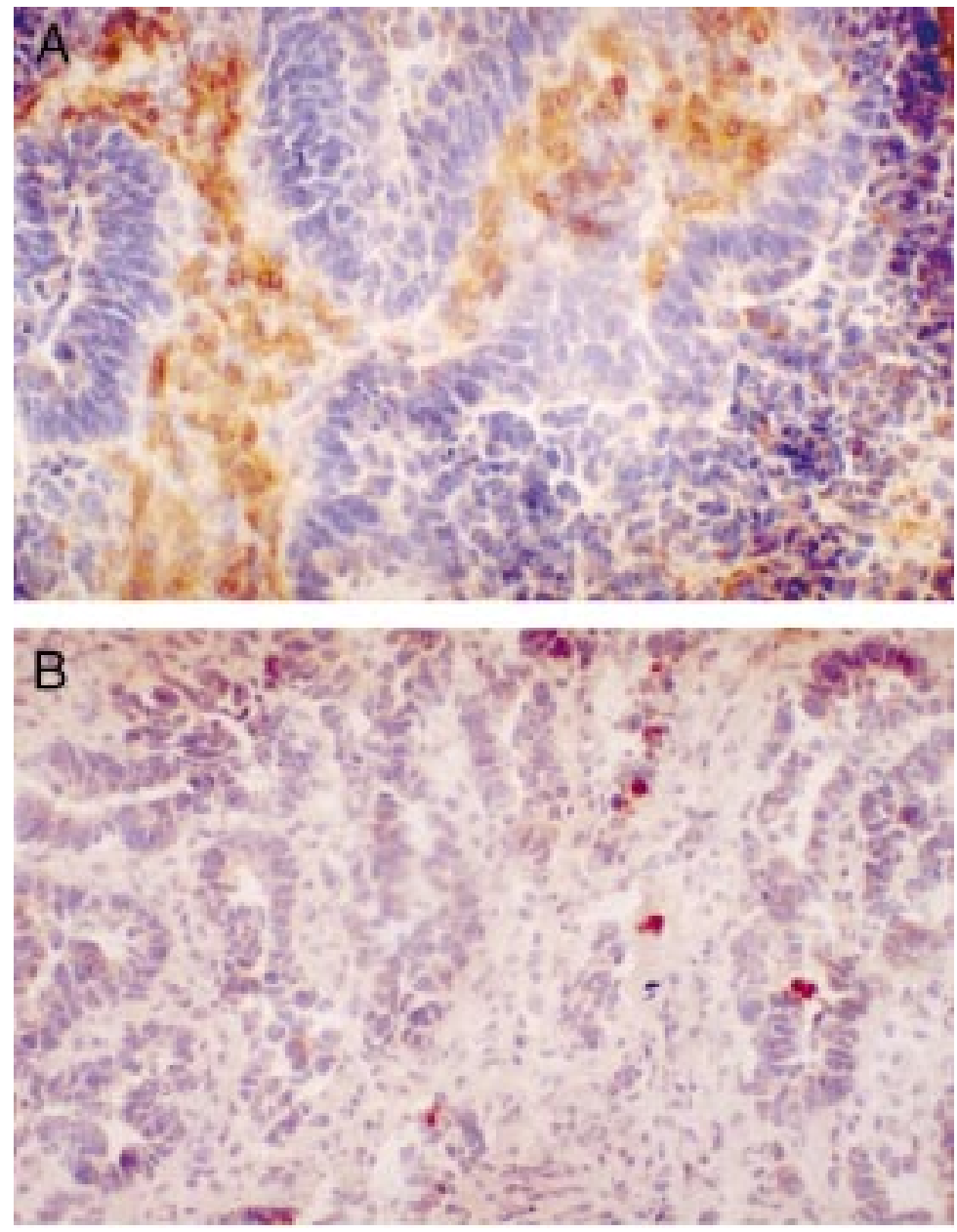

Figure 1 Infiltration of tumour centre by lymphocytes expressing CD25 in patients receiving $105 A D 7(A)$, and their matched controls (B). Original magnification $\times 200$.

used, and bound enzyme was visualised using 3,3'-diaminobenzidinetetrahydrochloride (DAB, Sigma Chemical Company). Slides were bathed in $0.59 \%$ copper sulphate, stained with haematoxylin, and dehydrated in graded concentrations of ethanol (figs 1 and 2). Sections of tonsil were used as a positive control, and similarly treated (data not shown).

IMAGE ANALYSIS

Sections were viewed under $\times 200$ magnification, and the image transferred by camera to an Apple Macintosh Quadra 660AV computer. Using the NIH image program it was possible to quantify the degree of brown staining relative to the blue haematoxylin background. The brown stain reflects expression of either the CD25 or $\mathrm{IgG}_{1}$ monoclonal antibody. Sections from normal bowel, and tumour edges and centre were each analysed in five different, randomly selected areas, and a mean pixel count obtained. Up to 15 individual pixel counts were therefore obtained for both CD25 and $\mathrm{IgG}_{1}$ for each tumour. Slides from both trial and control patients were analysed blindly by one observer.

TUMOUR INFILTRATING LYMPHOCYTES

Fresh tumour was available from 14 of the 19 patients immunised, and 31 controls. Approximately $1 \mathrm{~cm}^{3}$ of tumour was finely chopped in a Petri dish using a scalpel, and added to $10 \mathrm{ml}$ of $5 \%$ collagenase $\mathrm{H}$ (Sigma, $1 \mathrm{mg} / \mathrm{ml}$ ) in RPMI. After 10 minutes of incubation at $37^{\circ} \mathrm{C}$ for 10 minutes, released cells were collected, and again digested with collagenase $\mathrm{H}$. After centrifugation the cells were treated with DNAse (Sigma), washed by centrifugation, and resuspended in $\mathrm{RPMI} / 10 \%$ fetal calf serum (FCS).

Separation of lymphocytes from epithelial cells and red cells was achieved using a discontinuous Percoll gradient. A $5 \mathrm{ml}$ aliquot of disaggregated tumour suspension in RPMI $/ 10 \%$ FCS was added to the top of the gradient, and centrifuged for 30 minutes at $4^{\circ} \mathrm{C}$ and 2000 $\mathrm{rpm}$. The resultant band of lymphocytes was harvested from the interface between 1.055 and 1.077 densities, and washed in RPMI, prior to final resuspension in RPMI/10\% FCS.

\section{FLOW CYTOMETRY OF TUMOUR INFILTRATING} LYMPHOCYTES

Cells were stained with both CD25 (PE-Dako) and CD4 (FITC-Dako) monoclonal antibody. In addition the percentage of leucocytes in the tumour preparation was assessed by labelling the suspension with Leucogate (CD45/CD14, Becton Dickinson). An isotype matched labelled control was used to detect non-specific monoclonal antibody staining. After 30 minutes incubation with antibodies on ice, samples were centrifuged at $1200 \mathrm{rpm}$ for five minutes, washed twice with RPMI/1\% FCS, and fixed in $1 \%$ formaldehyde. Samples were analysed on a FACScan cytometer (Becton Dickinson).

Analysis of samples was performed blindly by one investigator (AG). Lymphocytes were gated originally using CD45. From this gate, the number of CD4+ lymphocytes expressing CD25 was then analysed, and expressed as a percentage of the total number of CD4+ lymphocytes.

\section{STATISTICAL ANALYSIS}

A two tailed Wilcoxon signed rank test was used to assess differences between trial and control patients in the paired analysis. A Mann-Whitney U test was used to test non-parametric unpaired data.

\section{Results}

There was no evidence of local reaction at the injection site, or evidence of systemic toxicity. Of the tumours removed, five were Dukes's stage $A$, seven stage $B$, six stage $C$, and one stage $\mathrm{D}$. All but two were moderately differentiated on routine histopathological analysis (table 1).

The control group for the flow cytometric analysis of disaggregated tumour consisted of cancers from 31 unimmunised patients. Three were Dukes's stage A, 15 stage B, 12 stage C, and one stage D. Five and one of the tumours were poorly and well differentiated, respectively, with the remainder classed as moderate.

Expression of CD25, as measured by immunohistochemistry, was higher in patients who had received 105AD7 (median 0.75 versus $0.50 ; p=0.06$; fig 3). Though this difference was not statistically significant, when the data 

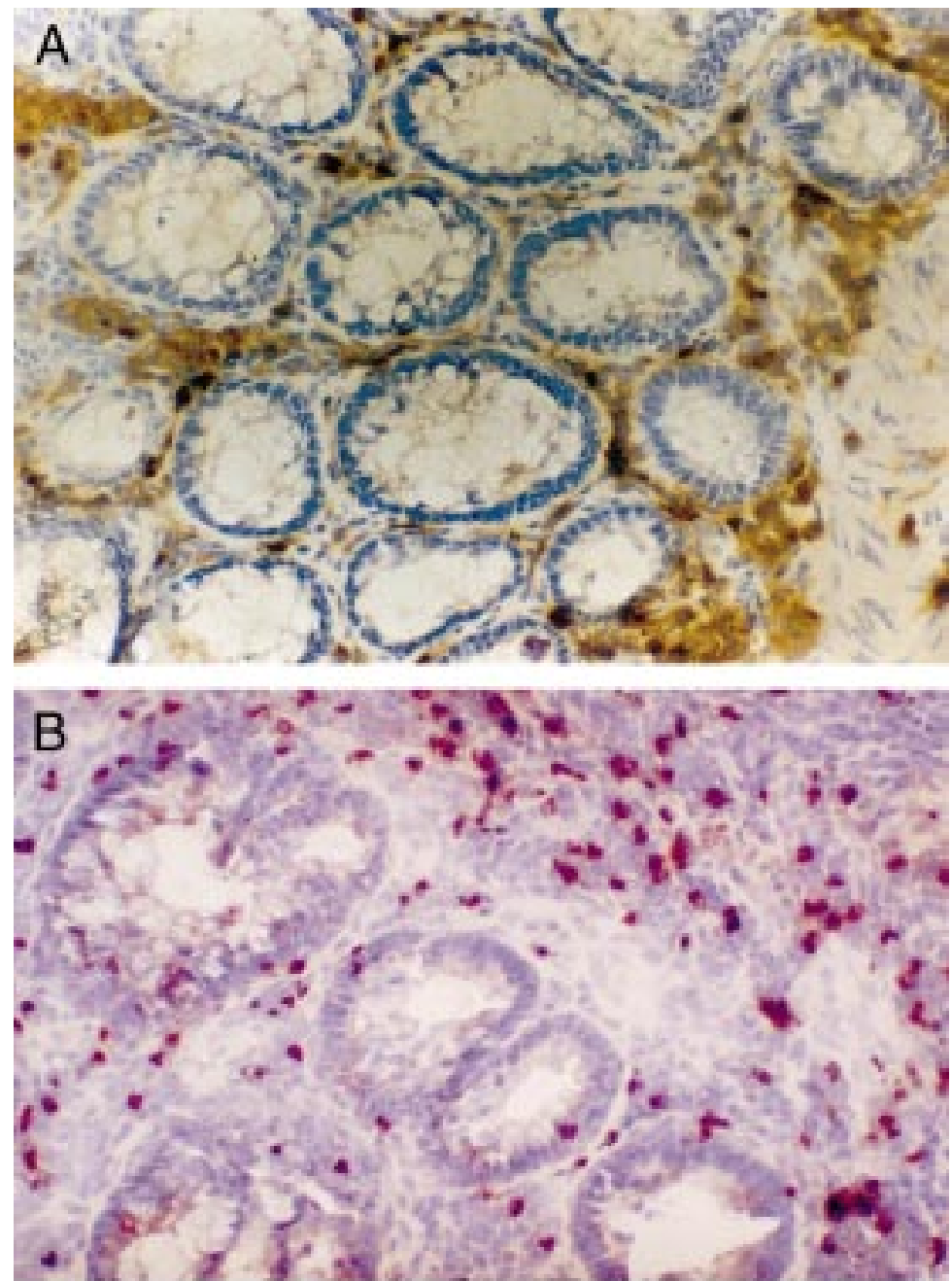

Figure 2 Infiltration by lymphocytes expressing CD25, at the tumour edge of trial patients who had received $105 A D 7(A)$, and their matched controls (B). Original magnification $\times$ 200.

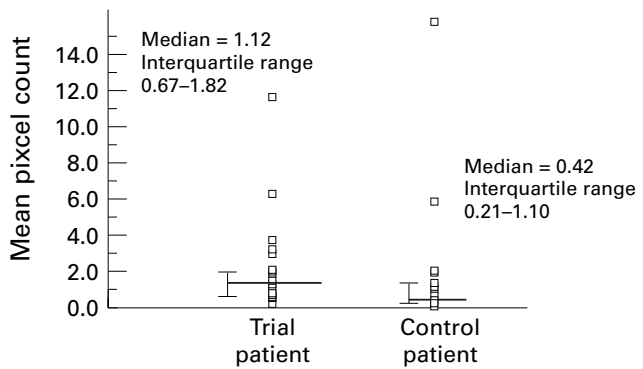

Figure 4 Tumour to normal bowel ratio of CD25 expressing lymphocytes.

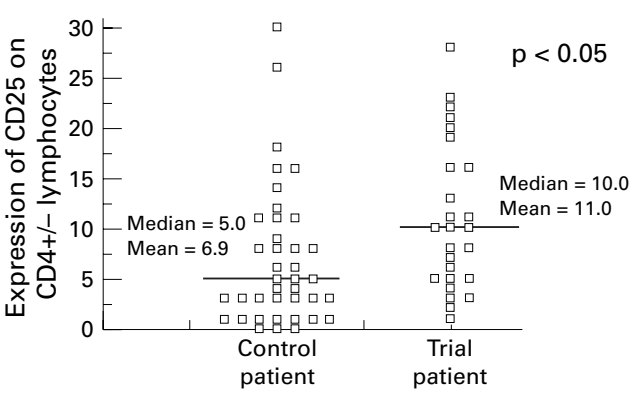

Figure 5 Percentage expression of CD25 on CD4+ and CD4- lymphocytes on tumour infiltrating lymphocytes, as measured by flow cytometry, in patients receiving 105 AD7 preoperatively, and unimmunised controls.

ratios of tumour to normal bowel: 0.61 (IQR 0.43 to 1.79 ) and 0.50 (IQR 0.26 to 0.82 ) in immunised and unimmunised patients respectively. This difference was not statistically significant.

Fresh tumours were available from 14 of the 19 patients who had received $105 \mathrm{AD} 7$ prior to surgery, and 31 control patients. Tumour disaggregation was performed for both groups as above. This separation is technically difficult, and analysis by flow cytometry is only possible if at least 1000 lymphocytes are isolated from the tumour digest. Results were therefore only obtainable in 13/14 trial patients, and 20/31 controls. Median percentage expression of CD25 on CD4+ and CD4- lymphocytes was $10 \%$ (IQR 5-16\%) in trial patients and 5\% (IQR 1.5-10.5\%) in controls $(p<0.05$; fig 5). In order to compensate for other variables, six fresh tumours from patients who had received 105AD7 were matched to six from the control group, according to site, stage, and differentiation of the tumour. Trial and control groups were also sex matched, and had comparable mean ages, 67.5 and 64.5 years respectively. All tumours were rectal and moderately differentiated, with equal numbers of Dukes's stage B and C tumours. Flow cytometric analysis of CD4+ lymphocytes infiltrating primary colorectal tumours of patients who had received $105 \mathrm{AD} 7$, showed that a median of $10.5 \%$ expressed the IL-2 receptor, compared with a figure of $6.5 \%$ in the unimmunised group $(\mathrm{p}=0.0044)$.

\section{Discussion}

It has been proposed that anti-idiotypic antibodies are taken up by dendritic cells in the skin, and presented to the immune system in association with class I and II major histocompatibility complex (MHC) glycoproteins, and

Figure 3 Immunohistochemical staining of tumour infiltrating lymphocytes expressing CD25 in trial and control patients, as measured by image analysis. 
costimulatory molecules. ${ }^{15}$ Cytotoxic and helper $\mathrm{T}$ cell responses capable of targeting the TAA that $105 \mathrm{AD} 7$ mimics may thus be elicited. Contact between primed $\mathrm{T}$ lymphocytes and tumour antigen induces synthesis of IL-2, and its receptor. Released IL-2 has been shown to promote activation, growth, and differentiation of a variety of leucocytes, including T lymphocytes, B cells, NK cells, and monocytes, and in addition further promote receptor formation. ${ }^{131617}$ The autocrine/ paracrine expression of this receptor therefore suggests lymphocyte activation. ${ }^{18} 19$ These results show that expression of the $\alpha$ subunit of the IL-2 receptor on lymphocytes infiltrating primary colorectal cancers is increased in patients immunised with the anti-idiotypic monoclonal antibody 105AD7, relative to well matched controls. This finding has been confirmed using two separate techniques, namely flow cytometry and immunohistochemistry.

Activation markers on TIL have similarly been measured using immunohistochemistry and flow cytometry. Expression of CD69 and HLA-DR is increased on lymphocytes infiltrating melanomas, following immunisation with autologous vaccine modified by the hapten DNP (dinitrophenyl).$^{20}$ However, less than $2 \%$ of infiltrating $\mathrm{T}$ lymphocytes in 11 of the 12 tumours analysed expressed CD25. This observation, the authors suggest, is a consequence of recruiting patients with advanced disease, who have been shown to have defective lymphokine production, reduced $\mathrm{CD} 3 \zeta$ chain and MHC molecule expression, as well as decreased cytotoxic $\mathrm{T}$ lymphocyte (CTL) activity.

While clearly two colour flow cytometry confirms the presence of CD25 on lymphocytes, it could be argued that the increased IL-2 receptor expression seen immunohistochemically could be due to its expression on tumour cells themselves. Weidmann et $a l^{11}$ showed that human squamous cell carcinomas of the head and neck expressed the $\beta$ subunit, as did gastric and renal carcinomas. Presence of the $\alpha$ subunit (CD25) was not however seen. A recent immunohistochemical analysis of 52 cryopreserved solid tumour specimens has confirmed that while the $\beta$ subunit is seen on tumour cells, the $\alpha$ subunit is not. ${ }^{22}$ These results suggest therefore that CD25 monoclonal antibody is labelling an IL-2 receptor present only on infiltrating lymphocytes. This is consistent with other authors who have confirmed the presence of CD25 immunohistochemically in cryopreserved tumour sections. ${ }^{23} 24$

It is encouraging to note that the same results were achieved by both flow cytometry and immunohistochemistry. The former analysis is prospective, allowing analysis of relatively large amounts of tumour, while the latter enables a topographical assessment of the tumour allowing a comparison between edge and centre. In our department we have cryopreserved a large number of tumour samples from unimmunised patients, enabling us to select a well matched control group for patients who have received $105 \mathrm{AD} 7$. Due to the fact that it is impossible to know stage and differentiation of disaggregated fresh tumours from unimmunised patients, prior to flow cytometry, it was necessary to analyse 31 patient tumours, before adequate matching could occur. The analysis was similarly performed blind. Trial patients were in addition well matched to controls according to the same five distinct variables (stage, differentiation, site, sex, and age) as used in the immunohistochemistry, and in each case the former had a higher percentage of CD4+ lymphocytes expressing CD25 than the latter.

Thirteen patients with advanced colorectal cancer have been immunised with 105AD7, as part of the previously described phase I study. ${ }^{7}$ Of these, six showed evidence of IL-2 production in their peripheral blood. In addition, immunohistochemical analysis of tumour sections from six patients immunised with 105AD7 preoperatively showed increased expression of CD25 in all samples relative to controls. ${ }^{11}$ Results outlined here in a different cohort of patients, confirm our original results, and show that patients immunised with $105 \mathrm{AD} 7$ prior to resection of their primary tumours have a higher tumour:normal ratio, and an increased number of CD25 expressing lymphocytes relative to controls, as measured by both immunohistochemistry and flow cytometry. This suggests a higher proportion of effector $\mathrm{T}$ cells at the tumour site in patients who have received the vaccine. While there is good evidence to suggest that TIL may have poor cytotoxic activity due to suppressor influences within the tumour environment, ${ }^{25} 26$ these results show that if primed lymphocytes are able to target antigen on the primary colorectal cancer, and become activated, then they may similarly be able to target antigen on circulating micrometastases. There then may be a role for a non-toxic vaccine as adjuvant therapy in patients with early stage disease.

The authors are grateful for the technical assistance of Mr Rob Moss and Miss Kate Bostock. They would also like to thank Professor RJC Steele and Mr NC Armitage for permission to recruit patients under their care to the study. This work was funded by the Cancer Research Campaign, 10 Cambridge Terrace, Regents Park, London, UK.

1 OPCS. Mortality statistics by cause: England and Wales 1992. Series DH2, number 20. London: HMSO, 1995.

2 Department of Health. On the state of the public health. The Annual Report of the Chief Medical Officer of the Department of Health. London: HMSO, 1991.

3 Maxwell-Armstrong CA, Durrant LG, Scholefield JH. Maxwell-Armstrong CA, Durrant LG, Scholefield
Colorectal cancer vaccines. Br 7 Surg 1998;85:149-54.

4 Maxwell-Armstrong CA, Durrant LG, Scholefield JH. Maxwell-Armstrong CA, Durrant LG, Scholefield JH.
Immune treatment for colorectal cancer. In: Recent advances in surgery. London: Churchill Livingstone, 1998: $97-111$

5 Austin EB, Robins RA, Durrant LG, et al. Human monoclonal anti-idiotypic antibody to the tumourassociated antibody 79IT/36. Immunology 1989;67:525-30.

6 Austin EB, Robins RA, Baldwin RW, et al. Induction of delayed hypersensitivity to human tumour cells with a human monoclonal anti-idiotypic antibody. $\mathrm{F}$ Natl Cancer Inst 1991;83:1245-8.

7 Denton GWL, Durrant LG, Hardcastle JD, et al. Clinical outcome of colorectal cancer patients treated with human monoclonal anti-idiotypic antibody. Int f Cancer 1995;57: $10-14$.

8 Robins RA, Denton GWL, Hardcastle JD, et al. Antitumor immune response and interleukin 2 production induced in colorectal cancer patients by immunisation with human monoclonal anti-idiotypic antibody. Cancer Res 1991;51: 5425-9. 
9 Jacob L, Somasundaram R, Smith W, et al. Cytotoxic T-cell clone against rectal carcinoma induced by stimulation of a patient's peripheral blood mononuclear cells with autolo-

10 Dourrant LG, Buckley TJD, Denton GWL, et al. Enhanced Durrant LG, Buckley TJD, Denton GWL, et al. Enhanced
cell-mediated tumour killing in patients immunized with human monoclonal antiidiotypic antibody 105AD7. Cancer Res 1994;54:4837-40

11 Buckley TJD, Robins RA, Durrant LC. Clinical evidence that the human monoclonal anti-idiotypic antibody 105AD7 delays tumor growth by stimulating anti-tumor T-cell responses. Hum Antibodies Hybridomas 1995;6:6872.

12 Maxwell-Armstrong CA, Buckley TJD, Durrant LC, et al. The anti-idiotypic monoclonal antibody 105AD7 increases lymphocyte infiltration within colorectal tumours [abstract]. Br F Surg 1996;83:1650.

13 Waldmann TA, Coldman CK, Robb RJ, et al. Expression of interleukin 2 receptors on activated human B cells. $\mathcal{f}$ Exp

14 Wang HM, Smith KA. The interleukin-2 receptor: functional consequences of its bimolecular structure. $\mathcal{F} \operatorname{Exp~Med}$ tional consequences

15 Rees ADM, Scoging A, Dobson N, et al. T cell activation by antiidiotypic antibody: mechanism of interaction with antigen-reactive T cells. Eur F Immunol 1987;17:197.

16 Henney CS, Kuribayashi K, Kern DE, et al. Interleukin-2 augments natural killer cell activity. Nature 1981;291:333 8.

17 Trinchieri G, Matsumoto-Kobayashi M, Clark SC, et al. Response of resting human peripheral blood natural killer cells to interleukin. F Exp Med 1984;160:1147-69.

18 Smith T. The interleukin-2 receptor. Annu Rev Cell Biol 1989;5:397-425.
19 Harel-Bellan A, Bertoglio J, Quillet A, et al. Interleukin-2 up-regulates its own receptor on a subset of human unprimed peripheral blood lymphocytes and triggers their proliferation. F Immunol 1986;136:2463-9.

20 Berd D, Maguire HC Jr, Mastrangelo MJ, et al. Activation markers on $\mathrm{T}$ cells infiltrating melanoma metastases after therapy with dinitrophenyl-conjugated vaccine Cancer Immunol Immunother 1994;39:141-7.

21 Weidmann E, Sacchi M, Plaisance S, et al. Receptors for interleukin-2 on human squamous cell carcinoma cell lines and tumor in situ. Cancer Res 1992;52:5963-70.

22 McMillan DN, Kernohan NB, Flett ME, et al. Interleukin-2 receptor expression and interleukin-2 localisation in human solid tumours in situ and in vitro: evidence for a direct role in the regulation of tumour cell proliferation. Int f Cancer 1995;60:766-72.

23 Hakansson L, Adell G, Boeryd B, et al. Infiltration of mononuclear inflammatory cells into primary colorectal carcinomas: an immunohistological analysis. Br $\mathrm{f}$ Cancer 1997;75:374-80.

24 Allen PM, Hogg N. Elevation of infiltrating mononuclear phagocytes in human colorectal tumors. F Natl Cancer Inst 1987;78:465.

25 Vose BM, Vanky F, Klein E. Human tumour-lymphocyte interaction in vitro. Comparison of the reactivity of tumour infiltrating blood and lymph-node lymphocytes with autologous tumour cells. Int f Cancer 1977;20:895-900.

26 Miescher S, Whiteside TL, Carrel S, et al. Functional properties of tumor-infiltrating and blood lymphocytes in patients with solid tumours: effects of tumor cells, and their supernatants on proliferative responses of lymphocytes. $\mathcal{F}$ Immunol 1986;136:1899-907. 